\title{
Caracterização do desenvolvimento motor e crescimento pondero-estatural de lactentes com tuberculose
}

\author{
Characterization of motor development and weight-height \\ growth of infants with tuberculosis
}

\author{
Mariana Fagundes Cinti $^{1}$, Raissa Felipe Pádua ${ }^{2}$, Cristina dos Santos Cardoso \\ de Sá $^{3}$
}

1.Graduanda de fisioterapia pela Universidade Federal de São Paulo, Santos-SP, Brasil.

2.Fisioterapeuta, Doutoranda do Programa Interdisciplinar em Ciências da Saúde da Universidade Federal de São Paulo, Santos-SP, Brasil.

3.Fisioterapeuta, Professora Doutora associada do Departamento de Ciências do Movimento Humano Universidade Federal de São Paulo - Campus Baixada Santista, Santos-SP, Brasil.

\section{Resumo}

Introdução. A tuberculose é uma doença infectocontagiosa; na infância é um evento sentinela causado pelo contato com adulto infectado, podendo acarretar atrasos no desenvolvimento motor, cognitivo, de linguagem e social do lactente. Fatores ambientais, culturais e sociais aos quais o lactente é exposto permitem seu desenvolvimento, portanto o isolamento e a visão da família e da sociedade sobre a tuberculose trazem contextos que impedem o relacionamento com outras crianças e outros adultos, podendo causar atrasos. Objetivo. Caracterizar o desenvolvimento motor de lactentes com tuberculose e o crescimento ponderoestatural, em comparação à lactentes sem tuberculose. Método. A avaliação do desenvolvimento motor foi realizada pela Escala Motora de Alberta, as medidas do crescimento pondero-estatural, pelo estadiômetro e balança do tipo pesa-lactente, e essas medidas foram comparadas à lactentes saudáveis. Resultados. A mesma porcentagem de lactentes com desenvolvimento motor suspeito foi observada em ambos os grupos; dois lactentes com tuberculose estão abaixo do peso esperado para idade e sexo. Conclusão. Os resultados não permitem afirmar que as alterações encontradas são decorrentes da enfermidade, pois, a região em que os lactentes e sua família estão inseridos, é de grande vulnerabilidade, e os fatores ambientais têm influência no desenvolvimento motor e antropométrico.

Unitermos. Tuberculose; Tuberculose Infantil; Desenvolvimento Infantil; Desenvolvimento Motor

\begin{abstract}
Introduction. Tuberculosis is an infectious disease. In childhood, it is a sentinel event caused by contact with an infected adult and may cause delays in the infant's motor, cognitive, language and social development. Environmental, cultural and social factors to which the infant is exposed allow its development, so the isolation and the family and society's view on tuberculosis bring contexts that prevent the relationship with other children and other adults, and may cause delays. Objective. To characterize motor development of infants with tuberculosis and weight-to-height growth compared to infants without tuberculosis. Method. The motor development was assessed by the Alberta Motor Scale, the measures of weight-height growth, the stadiometer and infant-weight scale, and these measurements were compared to healthy infants. Results. The same percentage of infants with suspected motor development was observed in both groups; two
\end{abstract}


tuberculosis infants are underweight for age and sex. Conclusion. The results do not allow us to state that the alterations found are due to the disease, since the region in which infants and their families are inserted is of great vulnerability, and environmental factors influence motor and anthropometric development

Keywords. Tuberculosis; Childhood Tuberculosis; Child Development; Motor Development

Trabalho realizado na Universidade Federal de São Paulo, Campus Baixada Santista. SantosSP, Brasil.

Conflito de interesse: não

Recebido em: 2018

Aceito em: 2018

Endereço para correspondência: Cristina SC Sá, R. Silva Jardim 136. Departamento de Ciências do Movimento Humano. Vila Mathias - Santos - SP. CEP: 11015-020. Email: cristina.sa@unifesp.br

\section{INTRODUÇÃO}

A tuberculose (TB) é uma doença infecciosa causada pela bactéria Mycobacterium Tuberculosis ou Bacilo de Koch, que afeta os pulmões, mas também pode acometer outros órgãos do corpo, como ossos, rins e meninges, levando a possíveis danos neurológicos ${ }^{1}$.

A epidemia de TB no Brasil tornou-se realidade na maior parte das cidades, sendo denominada "a praga dos pobres", devido a sua íntima relação com moradias insalubres com aglomeração de pessoas, falta de higiene e alimentação deficiente. Embora notória durante o século $X X$, a redução da mortalidade por TB não foi mantida nas últimas décadas ${ }^{2}$. Dentre as razões destacam-se a desigualdade social e seus determinantes, o advento da AIDS, a multirresistência do bacilo causador da enfermidade, o envelhecimento da população e os movimentos migratórios.

A presença de TB na infância é um evento sentinela causado pelo contato com adulto infectado. É comum seu 
aparecimento em menores de cinco anos de idade, e desenvolvem a doença, geralmente, dentro de um ano após a infecção, o que torna a tuberculose infantil um indicador da transmissão contínua da Mycobacterium tuberculosis na comunidade. Segundo a Organização Mundial da Saúde (2012), o Brasil tem incidência anual de 43 casos por 100 mil habitantes, taxa de 85 mil casos novos ao ano, dos quais $15 \%$ dos casos notificados acometem crianças menores de 15 anos. Recentemente, o Boletim Epidemiológico (2019), emitido pela Secretaria de Vigilância em Saúde, registrou que no ano de 2018 houve redução desses números, sendo diagnosticados 72.788 casos novos, correspondendo a um coeficiente de incidência de 34,8 casos por 100 mil habitantes ${ }^{3}$.

O diagnóstico da TB na infância ocorre pela avaliação clínico-radiológica associada à baciloscopia, cultura de escarro e recentemente por testes rápidos de biologia molecular, sempre que possível ${ }^{4}$. Porém, crianças menores de 10 anos apresentam especificidades que devem ser consideradas durante sua investigação diagnóstica. A forma pulmonar difere do adulto, pois costuma ser abacilífera ou paucibacilar, isto é, negativa ao exame bacteriológico, pelo reduzido número de bacilos nas lesões. Além disso, esse público, em geral, não é capaz de expectorar ${ }^{5}$. Na ausência de testes diagnósticos acessíveis e sensíveis, não baseados em amostras de escarro, diagnóstico confiável pode ser feito com um somatório de critérios que envolvem a história cuidadosa de exposição, quadro clínico-radiológico 
e investigações relevantes para a TB pulmonar e suspeita de TB extrapulmonar ${ }^{6}$.

Os sintomas observados em geral são: febre, anorexia, emagrecimento ou falta de ganho ponderal, astenia, irritabilidade e sudorese noturna. Alguns casos podem não apresentar sintomas respiratórios ou tosse. Em outros, os sintomas podem ser confundidos com pneumonia, asma brônquica, coqueluche ou bronquiolite. Muitas vezes, a suspeita de TB é feita em casos de pneumonia que não vêm apresentando melhora com o uso de antimicrobianos ${ }^{5,7}$. Outros sintomas como artralgia, eritema nodoso e conjuntivite flictenular, são sintomas pouco frequentes, e estão relacionados à hipersensibilidade ao Mycobacterium tuberculosis ${ }^{4,6}$.

A quimioprofilaxia primária é indicada para indivíduos ainda não infectados e empregada em recém-nascidos que coabitam com adultos bacilíferos. Desta forma, o RN não receberá a vacina BCG, logo ao nascer, mas uma outra abordagem ${ }^{4}$. O recém-nascido, que entrou em contato com o adulto bacilífero, deve receber quimioprofilaxia primária com isoniazida por três meses e, em seguida, realizar o derivado de proteína purificado (PPD). Caso apresente-se não reator, isto é, menor do que $5 \mathrm{~mm}$, deve-se suspender a isoniazida e aplicar a vacina Bacilo Calmette Guerin, conhecida como BCG intradérmica; em caso de o PPD ser reator, deve-se continuar o uso da isoniazida até seis meses de vida completos. Nestes casos, após o término, não há recomendação de vacinar com BCG. Entretanto, 
pela dificuldade em conseguir realizar 0 PPD, a quimioprofilaxia primária deve ter duração de seis meses e, ao término, há indicação de vacinação $\mathrm{BCG}^{8,9}$.

A Organização Mundial da Saúde (OMS) e o Fundo das Nações Unidas para a Infância (UNICEF) estimam que, a cada ano, morrem, no mundo, 2,5 milhões de crianças menores de cinco anos de idade por doenças que poderiam ter sido evitadas por ações de imunização ${ }^{10}$. O Ministério da Saúde (1994-2002) preconiza a vacinação a partir do nascimento. Desde que não tenha sido administrada na unidade neonatal, a vacina deve ser feita ao completar o primeiro mês de vida, em recém-nascidos, com pelo menos $2.000 \mathrm{~g}$ de peso. É indicada, principalmente, para prevenir as formas graves da tuberculose (miliar e meníngea) em crianças com menos de cinco anos de idade, mais frequentes em menores de um ano. Está indicada, também, o mais precocemente possível, para crianças HIV-positiva assintomática e filhos de mães HIV-positivas. Sendo contraindicada a indivíduos HIV-positivos sintomático. Portanto, a vacina é uma das principais estratégias para o controle da mortalidade por formas graves da infecção causada pelo Mycobacterium tuberculosis.

Alguns fatores têm sido apontados na literatura como obstáculos para a vacinação, tais como: baixa renda, residência em área rural, extremos de idade materna, maior número de filhos, maior ordem de nascimento, baixa escolaridade materna, trabalho materno fora do lar, maior número de moradores no domicílio, residência há menos de 
um ano na área, falta de conhecimento acerca das doenças imunopreveníveis, ausência de seguro-saúde e presença de doença na criança ${ }^{11}$.

O desenvolvimento infantil é um processo contínuo e complexo que se inicia no período intrauterino e segue de forma intensa nos primeiros anos de vida, sob influência de fatores genéticos, ambientais, culturais e sociais aos quais a criança está exposta ${ }^{12}$. Assim, a primeira infância, período de 0 a 6 anos de idade, é uma etapa fundamental para o desenvolvimento do ser humano ${ }^{13}$.

O desenvolvimento motor apresenta variações de movimentos e, a redução dessas variações, pode caracterizar-se por desenvolvimento suspeito. As mudanças que ocorrem durante os primeiros 12 meses de vida da criança são as mais importantes, por ocorrer grandes saltos evolutivos em curtos períodos de tempo. As modificações nos marcos do desenvolvimento típico são percebidas a cada mês, de maneira que as crianças podem ser inseridas em faixas etárias motoras por meio da presença de critérios como reações, reflexos, planos de movimento, padrões de movimento e aquisição de habilidades motoras ${ }^{14,15}$.

$O$ isolamento da criança, pela TB, traz efeitos inconvenientes para seu desenvolvimento de modo geral. Esse afastamento impede com que ela se socialize, troque informações e experiências, e brinque com outras crianças e até outros adultos. Por outro lado, a brincadeira está colocada como um dos princípios fundamentais, defendida como um direito e uma forma particular de expressão, 
pensamento, interação e comunicação entre as crianças $^{16}$. Além disso, a criança abacilífera e o estar em situação de isolamento é um momento cercado de emoções, sendo influenciado pela visão do próprio infectado sobre a sua doença, pela visão de sua família, da sociedade e pela história desses tipos de enfermidades. Isso porque, apesar das doenças transmissíveis serem tão antigas, ainda hoje é carregada de tabus e estigmas ${ }^{17}$.

É na infância que o indivíduo desenvolve recursos motores funcionais e cognitivos, que são de suma importância para seu desenvolvimento como ser biológico e social. Em razão disso, a identificação de possíveis riscos de atraso no desenvolvimento e crescimento da criança devem ser diagnosticados o mais precocemente possível para amenizar os impactos e realizar intervenção mais efetiva ${ }^{18}$. Mediante a isso, a observação do desenvolvimento sensório-motor é fundamental, pelas frequentes alterações transitórias, e também manifestação de deficiências graves $^{19}$. Com a detecção precoce dessas alterações, há a possibilidade de antecipação da estimulação necessária para o desenvolvimento global e estabelecimento de programas de prevenção de baixo custo $^{20}$.

Dessa forma este estudo objetivou caracterizar e comparar o desenvolvimento motor de lactentes com e sem tuberculose, assim como o crescimento pondero-estatural de lactentes com tuberculose. 


\section{MÉTODO}

\section{Amostra}

Trata-se de um estudo de caráter transversal de abordagem, aprovado pelo Comitê de Ética em pesquisa com Seres Humanos da UNIFESP, (número 1342/2018).

Foram recrutados 11 lactentes de 0 a 18 meses, de ambos os sexos e com diagnóstico de tuberculose atendidos na Seção Núcleo Integrado à Criança (SENIC), Santos-SP. Essa seção é ligada ao centro de referência de AIDS em Santos (CRAIDS/SANTOS), que tem um seguimento para o acompanhamento de crianças com exposição à tuberculose ou com diagnóstico de tuberculose. E ainda recrutados 11 lactentes na mesma faixa etária e sem o diagnóstico de tuberculose atendidos na Unidade básica de saúde da Vila Nova, Santos-SP, na qual os lactentes são acompanhados mensalmente até os seis meses de vida (período de amamentação exclusiva), e a cada dois meses até o primeiro ano de vida, e, posteriormente, a cada três meses seguindo a recomendação do Ministério da Saúde.

Foram incluídos no estudo lactentes entre 0 e 18 meses que foram expostos a tuberculose, não expostos ao vírus HIV, com idade gestacional acima de 37 semanas, que não apresentaram nenhum distúrbio neurológico e que os pais e/ou responsáveis estavam de acordo com participação do lactente no estudo. Contudo, não foram incluídos, lactentes que não estavam na faixa etária prédeterminada (0 a 18 meses), que não foram expostas a 
tuberculose, apresentavam algum tipo de distúrbio neurológico, foram expostos ao vírus HIV, com idade gestacional abaixo de 37 semanas, ou que os pais e/ou responsáveis não aceitaram a participação do lactente no estudo.

\section{Procedimento}

Realizou-se o levantamento dos lactentes acompanhados pelo SENIC, considerando os critérios de inclusão e exclusão. Em dia de consulta agendada com a pediatra do SENIC, os lactentes foram submetidos a avaliação do desenvolvimento motor por meio da Escala Motora Infantil de Alberta (Alberta Infant Motor Scale AIMS), desenvolvida em Alberta, no Canadá ${ }^{19,20}$, com tradução e adaptação para a língua portuguesa do Brasil e validação para a população brasileira ${ }^{21,22}$, por um único examinador devidamente treinado.

Esse instrumento de observação avalia o desenvolvimento dos recém-nascidos a termo e pré-termo, a partir de 37 semanas de idade gestacional até 18 meses de idade corrigida. Composta por 58 critérios motores, distribuídos em quatro subescalas que descrevem 0 desenvolvimento da movimentação espontânea e das habilidades motoras nas posturas prono (21 é a pontuação máxima), supino (9 é a pontuação máxima), sentado (12 é a pontuação máxima) e em pé (16 é a pontuação máxima). o examinador leva em consideração aspectos como descarga de peso corporal, equilíbrio e alinhamento 
postural, movimentações voluntárias e antigravitacional desempenhadas. De acordo com a escala, a postura é pontuada com ' 1 ' se observada ou com ' 0 ' se não observada. O escore bruto é obtido a partir da soma do escore em cada uma das subescalas, adicionado à idade do lactente e é convertido em percentil. Percentis são agrupados em categorias de desenvolvimento motor: abaixo de $5 \%$, considera-se que a criança tem desempenho motor anormal; entre $5 \%$ e $25 \%$, desempenho motor suspeito; e acima de $25 \%$, desempenho motor normal ${ }^{23}$.

Os dados referentes ao peso e estatura foram obtidos seguindo as recomendações da Sociedade Brasileira de Pediatria $(2009)^{24}$. A estatura foi aferida com o lactente deitado (despido e descalço), utilizando-se uma régua antropométrica sobre superfície plana, e o procedimento contou com a participação de dois examinadores (mãe/responsável e profissional). Já o peso foi obtido com a utilização de balança do tipo pesa-lactente, que possui grande precisão, com divisões de $10 \mathrm{~g}$ e capacidade de até $16 \mathrm{~kg}$, e para a aferição dessa medida a criança estava despida e descalça.

\section{Análise Estatística}

Realizou-se a análise descritiva dos dados: distribuição de frequência absoluta e percentílica para o percentil do desenvolvimento motor; e distribuição absoluta para as variáveis antropométricas: peso, altura e perímetro cefálico, e identificados se estes estavam ou não de acordo 
com os dados antropométricas do ministério da saúde para cada idade e sexo ${ }^{25}$.

Para comparar o desenvolvimento motor dos lactentes com e sem tuberculose foi realizado o teste $t$-student. Para comparar o crescimento pondero-estatural foi realizado 0 teste $t$ one comparando com os dados normativos da população brasileira. As análises foram realizadas no SPSS, versão 23. Nível de significância adotado foi de $5 \%$.

\section{RESULTADOS}

Foram recrutados 22 lactentes, sendo 11 lactentes identificados com tuberculose (grupo tuberculose), e outros 11 lactentes sem a doença (grupo controle). Em cada grupo havia lactentes com idade cronológica de quatro, seis, sete, nove e 12 meses.

No grupo dos lactentes com tuberculose foi observado que $18,2 \%$ (lactente 2 e lactente 5 ) apresentaram percentil entre $5-25 \%$, que caracteriza desenvolvimento suspeito Nota-se no grupo sem tuberculose que os lactentes com a mesma idade, não apresentaram percentil abaixo de $25 \%$. O restante do grupo tuberculose $(81,8 \%)$ apresentou desenvolvimento típico, com percentis maiores que 25\%, de acordo com a Escala Motora Infantil de Alberta (AIMS). Os lactentes 2 e 5 desse grupo compreendiam a idade cronológica de nove e 12 meses, respectivamente (Tabela 1). 
Tabela 1. Caracterização do desenvolvimento motor de lactentes com e se diagnóstico de tuberculose de acordo com a idade e sexo.

\begin{tabular}{c|cccccccc}
\hline $\begin{array}{c}\text { Lactentes } \\
\text { com } \\
\text { tuberculose }\end{array}$ & $\begin{array}{c}\text { Idade } \\
\text { (meses) }\end{array}$ & sexo & prono & supino & sentado & $\begin{array}{c}\text { em } \\
\text { pé }\end{array}$ & $\begin{array}{c}\text { escore } \\
\text { bruto }\end{array}$ & $\begin{array}{c}\text { percentil } \\
\text { AIMS } \\
\text { (\%) })\end{array}$ \\
\hline $\mathbf{1}$ & 4 & $\mathrm{M}$ & 5 & 4 & 4 & 2 & 15 & 50 \\
$\mathbf{2}$ & 9 & $\mathrm{M}$ & 14 & 9 & 10 & 2 & 35 & $10-25$ \\
$\mathbf{3}$ & 4 & $\mathrm{~F}$ & 5 & 5 & 3 & 2 & 15 & 50 \\
$\mathbf{4}$ & 7 & $\mathrm{~F}$ & 19 & 9 & 12 & 6 & 46 & 90 \\
$\mathbf{5}$ & 12 & $\mathrm{M}$ & 21 & 9 & 9 & 8 & 47 & $5-10$ \\
$\mathbf{6}$ & 12 & $\mathrm{~F}$ & 2 & 9 & 12 & 16 & 58 & 90 \\
$\mathbf{7}$ & 9 & $\mathrm{M}$ & 21 & 9 & 9 & 10 & 49 & $50-75$ \\
$\mathbf{8}$ & 6 & $\mathrm{~F}$ & 12 & 9 & 9 & 3 & 33 & $75-90$ \\
$\mathbf{9}$ & 6 & $\mathrm{M}$ & 13 & 9 & 6 & 2 & 30 & 75 \\
$\mathbf{1 0}$ & 12 & $\mathrm{M}$ & 21 & 9 & 12 & 13 & 55 & $50-75$ \\
$\mathbf{1 1}$ & 4 & $\mathrm{~F}$ & 6 & 6 & 4 & 3 & 19 & $50-75$ \\
\hline Lactentes & idade & sexo & prono & supino & sentado & em & escore & percentil \\
sem & (meses) & & & & & pé & bruto & AIMS \\
tuberculose & & & & & & & & (\%) \\
\hline $\mathbf{1}$ & 4 & $\mathrm{M}$ & 7 & 7 & 3 & 2 & 19 & 90 \\
$\mathbf{2}$ & 9 & $\mathrm{~F}$ & 20 & 9 & 12 & 6 & 47 & $50-75$ \\
\hline $\mathbf{3}$ & 4 & $\mathrm{M}$ & 6 & 7 & 5 & 3 & 21 & 25 \\
$\mathbf{4}$ & 7 & $\mathrm{~F}$ & 17 & 9 & 11 & 6 & 43 & 90 \\
$\mathbf{5}$ & 12 & $\mathrm{~F}$ & 19 & 9 & 12 & 12 & 52 & $25-50$ \\
$\mathbf{6}$ & 12 & $\mathrm{~F}$ & 21 & 9 & 12 & 16 & 58 & 90 \\
$\mathbf{7}$ & 9 & $\mathrm{~F}$ & 3 & 4 & 1 & 2 & 0 & $10-25$ \\
$\mathbf{8}$ & 6 & $\mathrm{~F}$ & 12 & 9 & 9 & 3 & 33 & 90 \\
$\mathbf{9}$ & 6 & $\mathrm{M}$ & 21 & 9 & 12 & 9 & 51 & 75 \\
$\mathbf{1 0}$ & 12 & $\mathrm{M}$ & 2 & 9 & 12 & 10 & 52 & $25-50$ \\
$\mathbf{1 1}$ & 4 & $\mathrm{~F}$ & 7 & 6 & 3 & 2 & 18 & $50-75$ \\
\hline & & & & & & & & \\
\hline
\end{tabular}

O grupo controle, de lactentes sem tuberculose, manifestou a mesma porcentagem $(18,2 \%)$ de lactentes com desenvolvimento suspeito. O lactente 3, com quatro meses de idade, apresentou percentil de $25 \%$ e o lactente 7 , com idade de nove meses, entre $10-25 \%$ na AIMS. Os lactentes do grupo tuberculose com a mesma idade e número de identificação, respectivamente, foram classificados com desenvolvimento motor normal. Os demais indivíduos do grupo foram classificados como desenvolvimento normal. 
Ao comparar os dois grupos não revelou diferença para o desenvolvimento motor.

As características do crescimento pondero-estatural do grupo dos lactentes com tuberculose foram avaliadas dispondo das mensurações de peso em gramas, altura em centímetros e perímetro cefálico (PC) em centímetros, e comparadas com os dados normativos da população brasileira, de acordo com as tabelas normativas para menores de 5 anos, aprovadas em 2006 pela OMS, e seguida pelo Ministério da Saúde desde então ${ }^{32}$. Essas medidas são avaliadas de acordo com a idade cronológica e o sexo do lactente. A Tabela 2 expõem os dados coletados no grupo de lactentes com tuberculose, contendo, também a idade e sexo, identificado como 1, para masculino e 2 para feminino, de cada lactente.

Tabela 2. Caracterização do peso (gramas-g), altura (centímetros-cm) e perímetro cefálico-pc (centímetros-cm) de acordo com a idade (meses-m) no dia da avaliação.

\begin{tabular}{c|c|c|c|c|c}
\hline $\begin{array}{c}\text { lactentes com } \\
\text { tuberculose }\end{array}$ & $\begin{array}{c}\text { Idade } \\
(\mathbf{m})\end{array}$ & sexo & $\begin{array}{c}\text { peso } \\
(\mathbf{g})\end{array}$ & $\begin{array}{c}\text { altura } \\
(\mathbf{c m})\end{array}$ & $\begin{array}{c}\text { pc } \\
(\mathbf{c m})\end{array}$ \\
\hline $\mathbf{1}$ & 4 & $\mathrm{M}$ & 6.300 & 64,5 & 41 \\
$\mathbf{2}$ & 9 & $\mathrm{M}$ & 9.970 & 68,5 & 44 \\
$\mathbf{3}$ & 4 & $\mathrm{~F}$ & 6.950 & 64,7 & 43 \\
$\mathbf{4}$ & 7,5 & $\mathrm{~F}$ & 8.000 & 70 & 42 \\
$\mathbf{5}$ & 12,25 & $\mathrm{M}$ & 10.000 & 72 & 43 \\
$\mathbf{6}$ & 12,25 & $\mathrm{~F}$ & 8.865 & 75 & 47 \\
$\mathbf{7}$ & 9,5 & $\mathrm{M}$ & 10.100 & 77 & 44 \\
$\mathbf{8}$ & 6,25 & $\mathrm{~F}$ & 4.450 & 68 & 42 \\
$\mathbf{9}$ & 6 & $\mathrm{M}$ & 5.840 & 64,5 & 44 \\
$\mathbf{1 0}$ & 12,25 & $\mathrm{M}$ & 10.310 & 74,2 & 44 \\
$\mathbf{1 1}$ & 4,25 & $\mathrm{~F}$ & 6.570 & 62 & 41 \\
\hline
\end{tabular}


Nessa amostra, dois lactentes $(18,2 \%)$ expostos à tuberculose (números 8 e 9), com a mesma idade cronológica de seis meses, apresentaram peso abaixo do recomendado pelo Ministério da Saúde. O peso/massa corporal deve estar entre os escores mínimo -2 (6.400 gramas) e máximo +2 (9.800 gramas) tolerável para a idade. O lactente número 8 demonstrou diminuição de $21,9 \%$ do peso mínimo esperado para a idade, colocando-o com escore abaixo de -3 (5.700 gramas) na curva, que indica peso muito abaixo para a idade. O número 9, também apresentou diminuição de peso, ficando 10,1\%, abaixo do peso mínimo, com escore entre - 3 e -2 da curva, indicando baixo peso. Os demais lactentes desse grupo permaneceram com escores dentro do esperado em sua idade.

Os resultados das medidas das alturas, dos lactentes expostos à tuberculose, demonstraram que o lactente 7 ultrapassou $0,5 \mathrm{~cm}$ da estatura esperada para a idade, ficando entre os escores +2 e +3 , indicando medidas adequadas. Os demais lactentes permaneceram com escores entre os esperados para a idade e sexo, e a média de altura encontrada nesse grupo foi de $62,9 \mathrm{~cm}$ no sexo feminino, e $70,1 \mathrm{~cm}$ no sexo masculino. E não houve diferença entre o grupo e os dados normativos.

As medidas de perímetro cefálico dos lactentes, comparadas às tabelas normativas, de acordo com os sexos e idade, apresentaram padrões entre os escores de medida esperados. 


\section{DISCUSSÃO}

O crescimento e o desenvolvimento do lactente são os principais indicadores de seu estado de saúde. Com isso, o Ministério da Saúde enfatiza o acompanhamento, desses lactentes por um pediatra, desde o nascimento até os 10 anos de idade. Com esse intuito, são realizadas consultas de puericultura, a fim de detectar, precocemente, possíveis alterações nesses indicadores ${ }^{26}$, e assim instalar condutas de prevenção de vacinação, alimentação, estimulação e cuidados gerais, como um processo contínuo de educação para a saúde.

Este estudo avaliou o desenvolvimento motor grosso e pondero-estatural de lactentes de quatro a 12 meses, expostos a tuberculose, em comparação a lactentes sem tuberculose, e às medidas normativas da população brasileira, respectivamente. Idades que se destacam por conterem marcos no desenvolvimento do lactente. $O$ desenvolvimento pode ser entendido como mudança ou expansão graduais, com surgimento e expansão das capacidades do indivíduo por meio do crescimento, da maturidade e do aprendizado, sendo um processo caracterizado pela progressão de estágios mais simples aos mais avançados na complexidade de movimentos ${ }^{27}$.

Segundo os dados coletados, o grupo de lactentes com tuberculose, apresentou baixa porcentagem (18,2\%) de lactentes com percentil entre $5-25 \%$ na escala AIMS, contabilizando dois com desenvolvimento suspeito. Em comparação ao grupo controle, foi notificado o mesmo 
número de lactentes com esse percentil na escala, porém com idades e sexos diferentes. Esses dados, obtidos com a AIMS, não permitem corroborar que, as alterações fisiológicas que a doença pulmonar causa no lactente abacilífero, podem prejudicar, de forma direta ou indireta, seu desenvolvimento motor grosso.

As medidas de estatura e perímetro cefálico dos lactentes com tuberculose manteve-se dentro dos escores esperados, com apenas um lactente $0,5 \mathrm{~cm}$ acima do esperado para sua altura. Dois lactentes apresentaram peso abaixo do esperado, com diminuição de, em média, $16 \%$ do peso mínimo para a idade e sexo. Esses pequenos percentuais de alterações antropométricas, não podem ser apontadas diretamente, como complicações devido a tuberculose. Mediante ao pequeno número de lactentes que apresentaram alterações significantes, e que existem outros aspectos, que influenciam no crescimento do indivíduo, como fatores intrínsecos, como o potencial genético e metabólico; e fatores extrínsecos, dentre eles, a alimentação, saúde, higiene, habitação e cuidados gerais com o lactente ${ }^{28}$.

Além disso, o baixo peso ao nascer, baixa escolaridade materna, gemelaridade, intervalo intergestacional curto (inferior a dois anos), criança indesejável, desmame precoce, mortalidade em crianças menores de 5 anos na família, condições inadequadas de moradia, baixa renda e desestruturação familiar, são considerados fatores de risco 
para um déficit no crescimento e desenvolvimento do lactente.

Por ser composto por características biopsicossociais, - ser humano sofre influências demográficas, socioeconômicas, culturais, psicológicas e ambientais, que interferem em seu desenvolvimento motor e cognitivo, desde a primeira infância. As condições ambientais, como os níveis de estimulação cognitiva, que o lactente recebe no lar, bem como a rotina da família e o status cultural, educacional e socioeconômico, podem afetar diretamente seu desenvolvimento. Bem como fatores psicossociais, a pobreza e os problemas relacionados a ela, como a baixa escolaridade dos progenitores, má nutrição e condições precárias de moradia, influenciam negativamente nesse desenvolvimento, sendo mais frequentes nas populações de baixa renda ${ }^{29}$, como é o caso da população avaliada no presente estudo.

Essas precárias condições de vida, assemelha-se às condições territoriais em que as famílias dos lactentes avaliados no presente estudo estão inseridas. A região em que residem, de alta vulnerabilidade, pode acarretar em déficits no desenvolvimento motor e pondero-estatural, não apresentando grandes correlações com aspectos, meramente biológicos. Mas sim, por escassa estimulação. Apesar das famílias não terem sido avaliadas, por meio de questionários socioeconômicos e socioculturais, é possível pressupor que as alterações no desenvolvimento motor e pondero-estaturais, podem estar interligadas com as 
condições socioeconômicas e redes de apoio em que o lactente e sua família têm acesso e estão inseridos.

\section{CONCLUSÃO}

Apesar de alguns lactentes com tuberculose apresentarem desenvolvimento motor suspeito, não é possível afirmar que essa alteração seja decorrente da enfermidade. Visto que o grupo controle, de lactentes sem tuberculose também apresentou. O mesmo ocorre em relação ao crescimento pondero-estatural dos lactentes com tuberculose.

Além da baixa evidência, essas variáveis avaliadas no presente estudo, são interdependentes de fatores ambientais, entre eles, socioeconômicos, culturais e demográficos, de que o lactente e sua família estão inseridos, que não foram avaliados pelo estudo. Por essa razão, seria interessante a aplicação de um questionário que abrangesse esses fatores ambientais, para avaliar 0 lactente levando em conta todas as suas dimensões, e assim, tornar a pesquisa mais completa.

\section{REREFÊNCIAS}

1.Tuberculose (endereço na internet). Secretaria da Saúde Governo do Estado do Paraná (acessado em: 13/11/2017). Disponível em: http://www.saude.pr.gov.br/modules/conteudo/conteudo.php?conteu do $=939$

2.Ruffino-Netto A. Tuberculose: a calamidade negligenciada. Rev Soc Bras Med Trop 2002;35:51-8. http://dx.doi.org/10.1590/S003786822002000100010

3.Secretaria de Vigilância em Saúde - Ministério da Saúde. Implantação do Plano Nacional pelo Fim da Tuberculose como 
Problema de Saúde Pública no Brasil: primeiros passos rumo ao alcance das metas. Boletim Epidemiológico 2018;49(11):1-18.

4.Alves R, Saar SMA, Sant'anna CC. Principais dúvidas dos pediatras sobre tuberculose em crianças e adolescentes. Resid Pediatr 2018;8:27-37. https://doi.org/10.25060/residpediatr

5.Ministério da Saúde, Secretaria de Vigilância em Saúde, Departamento de Vigilância Epidemiológica. Manual de recomendações para o controle da tuberculose no Brasil. Brasília: Ministério da Saúde, 2011.

6.Sant'anna CC, Mourgues LV, Ferrero F, Balanzat AM. Diagnóstico e terapêutica da tuberculose infantil: uma visão atualizada de um antigo problema. J Pediatr 2002;78(Supl2):205-14. http://dx.doi.org/10.1590/S0021-75572002000800011

7.Sant'anna CC. Pulmonary tuberculosis in adolescents: radiographic features. Int J Tuberc Lung Dis 2009;13:1566-8.

8.Sant'anna CC. Quimioprofilaxia da tuberculose. Pulmão RJ 2007; $16: 82-5$.

9.Sant'anna CC, Loboguerrero MA. Tuberculose em Crianças e Jovens. São Paulo: Editora Atheneu; 2015.

10.Fundo das Nações Unidas para a Infância. Situação mundial da infância 2008: sobrevivência infantil. Brasília: Fundo das Nações Unidas para a Infância; 2007.

11. Molina AC, Godoy I, Carvalho LR, Caldas JAL. Situação vacinal infantil e características individuais e familiares do interior de São Paulo. Rev Acta Sci Healh Sci 2007;29:99-106. http://dx.doi.org/10.4025/actascihealthsci.1077

12. Rugolo LMSS. Importância da monitorização do desenvolvimento em recém-nascidos prematuros. Rev Paul Pediatr 2012;30:460-1. http://dx.doi.org/10.1590/s0103-05822012000400001

13. Delaney L, Doyle O. Socioeconomic differences in early childhood time preferences. J Econom Psychol 2012;33:237-47. https://doi.org/10.1016/j.joep.2011.08.010

14.Flehming I. Atlas do desenvolvimento normal e seus desvios no lactente: Diagnóstico e Tratamento precoce do Nascimento até o $18^{\circ}$ mês. São Paulo: Atheneu, 2005.

15. Castilho-Weinert LV, Lopes HS. Sistema de apoio ao diagnóstico em fisioterapia neuropediátrica. Rev Fisioterap Ser 2010;5:37-42.

16. Ministério da Educação e do Desporto, Secretaria de Educação Fundamental. Referencial Curricular Nacional para a Educação Infantil. Brasília: MEC, 1998.

17. Gir E, Moriya T. O papel da enfermeira, da auxiliar de enfermagem e do atendente de enfermagem na assistência ao paciente em isolamento de doenças transmissíveis. Rev Esc Enferm USP 1991;25:271-86. http://dx.doi.org/10.1590/0080$\underline{6234199102500300271}$

18. Blawn-Hospers $\mathrm{CH}$, Hadders-Algra M. A systematic review of the effects of early intervation on motor development. Develop Med Child Neurol 2005;47:421-32.

http://dx.doi.org/10.1017/s0012162205000824 
19.Contesini LA, Sinhorini IR, Takiuchi N. Desenvolvimento global e de linguagem em crianças nascidas grandes para idade gestacional: relato de três casos. Rev CEFAC 2006;8:272-80.

20.Piper MC, Pinnell LE, Darrah J, Maguire T, Byrne PJ. Construction and validation of the Alberta Infant Motor Scale (AIMS). Can J Public Health 1992;83:46-50.

21.Piper MC, Darrah J. Motor assessment of the developing infant. Alberta: Saunders; 1994.

22.Saccani R, Valentini NC. Cross-cultural analysis of the motor development of Brazilian, Greek and Canadian infants assessed with the Alberta Infant Motor Scale. Rev Paul Pediatr 2013;31:350-8. http://dx.doi.org/10.1590/S0103-05822013000300012

23.Saccani R, Valentini NC, Pereira KRG. New Brazilian developmental curves and reference values for the Alberta infant motor scale. Infant Behav Dev 2016;45:38-46.

http://dx.doi.org/10.1016/j.infbeh.2016.09.002

24.Valentini NC, Saccani R. Brazilian validation of the Alberta Infant Motor Scale. Phys Ther 2012;93:440-7. http://dx.doi.org/10.2522/ptj.20110036

25.Sociedade Brasileira de Pediatria. Avaliação nutricional da criança e do adolescente - Manual de Orientação / Sociedade Brasileira de Pediatria. Departamento de Nutrologia. - São Paulo: Sociedade Brasileira de Pediatria. Departamento de Nutrologia, 2009.

26. Ministério da Saúde. Orientações para a coleta e análise de dados antropométricos em serviços de saúde: Norma Técnica do Sistema de Vigilância Alimentar e Nutricional-SISVAN. Brasília: Ministério da Saúde, 2011.

27. Chaves CMP, Lima FET, Mendonça LBA, Custódio IL, Matias EO. Avaliação do crescimento e desenvolvimento de crianças institucionalizadas. Rev Bras Enferm 2013;66:668-74.

http://dx.doi.org/10.1590/S0034-71672013000500005

28.Ministério da Saúde. Secretaria de Políticas de Saúde. Departamento de Atenção Básica. Saúde da criança: acompanhamento do crescimento e desenvolvimento infantil. Brasília: Ministério da Saúde, 2002.

29.Tella P, Piccolo LR, Rangel ML, Rohde LA, Polanczyk GV, Miguel EC, et al. Socioeconomic diversities and infant development at 6 to 9 months in a poverty area of São Paulo, Brazil. Trends Psychiatr Psychother 2018;40:232-40.http://dx.doi.org/10.1590/2237-6089-

2017-0008 